\title{
Características de la pubertad de niñas escolares de la Región Metropolitana
}

\author{
Dra. Raquel Burrows A.; T.M. Laura Leiva B.; Dra. Ada Maurieci N.; \\ St. Abraham Zvaighaft F.; Dr. Santiago Muzzo B. ${ }^{1}$ \\ Puberal characteristics of school age chilean girls
}

\begin{abstract}
The characteristics of puberal development were observed in a transversal study of 2328 school age temales. This sample was representative of girls from elementary schools of metropolitan Santiago belonging to high. Ineditum and low socioeconomic level (SEL). The age at initiation of puberty ranged from 93 to 183 months for mamary development and for pubic hair from 10010183 months. A significant chronological difference in the initiation of breast and pubic hair development was found between subjects belonging to medium and low SFL: the mamary development ocurring at an tarlier agc, compared with high SEL: 123.7 and 122.4 vs 128.6 and 126.8 month of age respectivelly. It is possible that environmental and genetic factors may be involved in this findings.

(Key words: puberal tharacteristics, girls, elementary school).
\end{abstract}

La evaluación del crecimiento y desarrollo de un suje to, es un buen indicador de su estado de salud. Una alteración de éstos procesos, puede indicar la presencia de afecciones como deficit de nutrientes, alteraciones genéticas, endocrinas $y$ otras enfermedades sistémicas. $1 \cdot 3$

La pubertad, es uno de los procesos de desarrollo durante la adolescencia en la que ocurren los cambios físicos, a consecuencia de cambios hormonales, que transforman al niño en un adulto con capacidad reproductiva.4-7 La pubertad se produce normalmente con una secuencia, existiendo variaciones fisjológicas de un individuo a otro. Asi, en adolescentes europeas se han pesquisado signos iniciales de desarrollo puberal entre $\operatorname{los} 8$ y $\operatorname{los} 15$ años.8.9 Estas amplias variaciones deben ser conocidas para detectar eventuales trastornos.

Se ha demostrado que influyen factores genéticos y ambientales en la edad de inicio y en la velocidad con que progresan los eventos puberales. ${ }^{10-12}$ El déficit global de nutrientes, especialmente calórico, es un factor ambiental que pro-

1. Unidad de Endocrinología. Instituto de Nutrición y Tecnología de Ios Alimentos, Universidad de Chile. duce un franco retraso de la pubertad y altera la pubertad que ya está en curso.13-15 Se ha demostrado retraso de la pubertad en escolares venezolanos de estratos socioeconómicos más bajos ${ }^{56} y$ presentación más temprana de la pubertad en la generación actual al compararla con generaciones anteriores, no pudiendo establecerse la real influencia de los factores genéticos y ambientales. Por otro lado, se ha descrito que factores genéticos pueden jugar un rol importante: las adolescentes asiáticas tienen su desarrollo puberal más temprano que las europeas. ${ }^{17}$ Dentro de un mismo país existen variaciones en la edad de presentación del desarrollo mamario, vello púbico y de la menarquia en adolescentes que viven en diferentes regiones lo que podría estar relacionado con factores nutricionales climáticos, raciales y socioeconómicos.9.18, 19.23 En este estudio se describe el desarrollo mamatio $y$ puberal alcanzado en niñas chilenas $y$ se le relaciona con algunas variables socioecnómicas.

\section{MATERIAL Y METODOS}

Se readizo un estudio transversal on una muestra tormada por 2.328 escolares de sexo femenino de 8 a 15 años. obtenidas del año 1982 a 1984 , la muestra fue aleatoria $y$ representativa de escolares de ensenanza 
básjca de la Región Metropolitana. Previa autorización por escrito de los padres, se realizó en cada escolar un examen físico, para evaluar el grado de desarrollo mamario (M) y velto púbico (P) de acuerdo a los cinco estadios ( 1 a 5 ) descritos por Tanneer. ${ }^{B}$ Esta cvaluación fue hecha por cos pediatras previamente entrenados. El nivel socioeconómico (NSE) se evaluó por el método Graffar modificado, 20 a través de una encuesta personal del apoderado, que lo clasifica en una escala del 1 al 6 . donde $I$ es el nivel alto y 6 el de miseria.

Los resultados se analizaron estadísticamente por el método de próbito, ${ }^{22}$ prueba $t$ de Student, análisis de varianza (prueba F) y $X^{2}$.

\section{RESULTADOS}

La tabla 1 muestra el estadio de desarrollo mamario alcanzado por 2.307 escolares de 8 a 15 años, según grupo etario. Se observa que a los 10 años de edad $40,5 \%$ de las escolares ya tienen algún grado de desarrollo mamario y a los 13 años $96,9 \%$ de las adolescentes.

La tabla 2 muestra el estadio de desarrollo de vello púbico por grupo etario de las escolares. A

Tabla 1.

Desarmollo mamario en 2.307 escolares de sexo femenino de Santiago, por grupo etario

\begin{tabular}{|c|c|c|c|c|c|c|c|c|c|c|c|c|}
\hline \multirow{2}{*}{$\begin{array}{l}\text { EDAD } \\
\text { (años) }\end{array}$} & \multicolumn{2}{|c|}{$\mathrm{Ml}^{*}$} & \multicolumn{2}{|c|}{ M2* } & \multicolumn{2}{|c|}{$\mathrm{M} 3 *$} & \multicolumn{2}{|c|}{ M4* } & \multicolumn{2}{|c|}{ M5* } & \multicolumn{2}{|c|}{ TOTAL } \\
\hline & $N$ & $\%$ & $\mathrm{~N}$ & $\%$ & $N$ & \% & $\mathrm{N}$ & $\%$ & $\mathbf{N}$ & $\%$ & $\mathbf{N}$ & \% \\
\hline$B .0$ & 100 & 96,2 & 4 & 3,8 & 0 & 0,0 & 0 & 0.0 & 0 & 0,0 & 104 & 100,0 \\
\hline $8,5-9,0$ & 100 & 93,5 & 6 & 5.6 & 1 & 0.9 & 0 & 0,0 & 0 & 0,0 & 107 & 100,0 \\
\hline $9,0-9,5$ & 72 & 82,9 & 11 & 12.6 & $\underline{1}$ & 3.4 & I & 1,1 & 0 & 0,0 & 87 & 100,0 \\
\hline $9,5 \cdot 10,0$ & 91 & $77.8-$ & 23 & 19,7 & 2 & 1.7 & 0 & 0,0 & 1 & 0,8 & 117 & 100,0 \\
\hline $10,0-10,5$ & 66 & $59.5-$ & 33 & 29.7 & 12 & 10,8 & 0 & 0,0 & 0 & 0,0 & 111 & 100,0 \\
\hline $10,5-11,0$ & 50 & 38.6 & 48 & 36,9 & 25 & 19,2 & 5 & 3,8 & 2 & 1,5 & 130 & 100,0 \\
\hline $11,0-11,5$ & 84 & 32,2 & 90 & 34,5 & 52 & 19,9 & $2\}$ & 10,3 & 8 & 3,1 & 261 & 100,0 \\
\hline $11,5-12,0$ & 39 & 16.5 & 82 & 34,8 & 68 & $2 B, 8$ & 38 & 16,1 & 9 & 3,8 & 236 & 100.0 \\
\hline $12,0-12,5$ & 38 & 14.4 & 61 & 23,1 & 85 & 32,2 & 60 & 22,7 & 20 & 7,6 & 254 & 100,0 \\
\hline $12,5-13,0$ & 13 & 5,6 & 44 & 18,9 & 77 & 33,0 & 66 & 28.3 & $33-$ & 14,2 & 233 & 100,0 \\
\hline $13,0-13,5$ & 7 & 2,8 & 31 & 12,2 & 62 & 24,3 & 92 & 36,0 & 63 & 24,7 & 255 & 100,0 \\
\hline $13,5-14.0$ & 7 & 3,1 & 10 & 4,5 & 41 & 18.3 & 92 & 41,1 & 74 & 33,0 & 224 & 100,0 \\
\hline $14,0-14,5$ & 0 & 0,0 & 1 & 0,9 & 25 & 22.3 & 50 & 44,7 & 36 & 32,1 & 112 & 100,0 \\
\hline $14.5-15,0$ & 0 & 0,0 & 2 & 4,9 & 6 & 14,6 & 20 & 48,8 & 13 & 31.7 & 41 & 100,0 \\
\hline $15,0-15.5$ & 1 & 4,0 & 1 & 4,0 & 2 & 8,0 & 13 & 52,0 & 8 & 32,0 & 25 & 100,0 \\
\hline Total & 668 & & 447 & & 461 & & 464 & & 267 & & 2307 & \\
\hline
\end{tabular}

$M^{*}=$ Lsiadio de desarrolto mamario

Tabla 2.

Desarrollo de vello púbico en 2.328 escolares de sexo femenino de Santiago, por grupo etario.

\begin{tabular}{|c|c|c|c|c|c|c|c|c|c|c|c|c|}
\hline \multirow{2}{*}{$\begin{array}{l}\text { EDAD } \\
(a \bar{n} \emptyset s)\end{array}$} & \multicolumn{2}{|c|}{$\mathrm{Pl}^{*}$} & \multicolumn{2}{|c|}{$\mathbf{P} 2^{*}$} & \multicolumn{2}{|c|}{ P3* } & \multicolumn{2}{|c|}{$\mathrm{P4}^{*}$} & \multicolumn{2}{|c|}{ PS* } & \multicolumn{2}{|c|}{ TOTAL } \\
\hline & $\mathbf{N}$ & $\%$ & $\mathbf{N}$ & $\%$ & $\mathrm{~V}$ & 16 & $N$ & $\%$ & $\mathbf{N}$ & $\%$ & $N$ & $\%$ \\
\hline $8,0-8,5$ & 106 & 99,1 & 1 & 0.9 & 0 & 0,0 & 0 & 0,0 & 0 & 0.0 & 107 & 100,0 \\
\hline $8,5-9,0$ & 106 & 98,1 & & 1,9 & 0 & $\emptyset, 0$ & 0 & 0,0 & 0 & 0.0 & 108 & 100,0 \\
\hline $9.0-9,5$ & 77 & 88,6 & 9 & 10,3 & I & 1,1 & 0 & 0,0 & D & 0,0 & 87 & 100,0 \\
\hline $9,5-10,0$ & 107 & 91,5 & 8 & 6.7 & $\mathbf{I}$ & 0,9 & 0 & 0,0 & 1 & 0,9 & 117 & 100,0 \\
\hline $10,0-10,5$ & 84 & 75,0 & 21 & 18,8 & 7 & 6,2 & 0 & 0,0 & 0 & 0,0 & 112 & $100_{2} 0$ \\
\hline $10.5-11.0$ & 74 & 56,0 & 39 & 29,5 & 15 & 11,4 & 3 & 2,3 & 1 & 1,5 & 132 & 100,0 \\
\hline $11,0-11,5$ & 92 & 35,1 & 111 & 42,4 & 42 & 16,0 & 13 & 5,0 & 4 & 1,5 & 262 & 100,0 \\
\hline $11,5-12,0$ & 53 & 23,4 & 92 & 39.2 & 56 & 23,8 & 31 & 13.2 & ] & 0,4 & 235 & 100,0 \\
\hline $12,0-12,5$ & 33 & 11,7 & 97 & 34,5 & 90 & 32,0 & 55 & 19.6 & 6 & 2,1 & 281 & 100.0 \\
\hline $12,5-13,0$ & 13 & 5,7 & 60 & 26,2 & 76 & 33,2 & 75 & 32.7 & 5 & 2,2 & 229 & 100,0 \\
\hline $13,0 \sim 13,5$ & $\tau$ & 2,8 & 41 & 16,1 & 77 & 30,3 & 103 & 40,6 & 26 & 10,2 & 254 & 100,0 \\
\hline $13,5-14,0$ & 3 & 1,3 & 15 & 6,7 & 59 & 26.3 & 121 & 54,1 & 26 & 11,6 & 224 & 100,0 \\
\hline $14,0-14,5$ & 0 & 0,0 & 4 & 3.5 & 27 & 23.7 & 56 & 49,1 & 27 & 23,7 & 114 & 100,0 \\
\hline $14,5-15,0$ & 0 & 0,0 & $2^{\circ}$ & 5,9 & 6 & 14,6 & 23 & 56,1 & 10 & 25,1 & 41 & 100,0 \\
\hline I 5,0 & 0 & 0,0 & 1 & 4,0 & 5 & 10,0 & 12 & 48,0 & 7 & 28.0 & 25 & 100,0 \\
\hline TOT AL & 757 & & 503 & & 462 & & 492 & & 114 & & $232 \mathrm{~B}$ & \\
\hline
\end{tabular}

$\mathbf{P}^{*}=$ Vcho Púbico. 
los 10 años existen $25 \%$ de niñas con desarrollo de vello púbico.

Los rasgos de edad para las etapas injciales y finales de desarrollo puberal fueron de 96 a 183 meses para $\mathrm{M} 2,100$ a 183 meses para $\mathrm{P} 2$ y 117 a 185 meses para M5 y P5.

Al analizar la edad de promedio de inicjo del

Tabla 3.

Edad promedio (meses) de inicio de desarrollo mamario $y$ de vello púbico en escolares de sexo femenina por nivel socioeconómico

\begin{tabular}{|c|c|c|c|}
\hline $\begin{array}{c}\text { Nivel Sochestonommico } \\
\text { (Gratiac) }\end{array}$ & $\begin{array}{c}\text { Giado de Inear resllo } \\
\mathrm{N}_{2}\end{array}$ & $\begin{array}{c}\text { Puberal i] anner! } \\
\qquad 2\end{array}$ & $\mathrm{pb}$ \\
\hline $1+2$ & $\begin{array}{c}126.78 \pm 14.10^{9} \\
(180)\end{array}$ & $\begin{array}{c}127.73 \pm 12.52 \\
(178)\end{array}$ & NS \\
\hline 3 & $\begin{array}{c}123.77 \pm 17.01 \\
(1] 2)\end{array}$ & $\begin{array}{c}128.65 \pm 12.30 \\
4387 ?\end{array}$ & $<0.001$ \\
\hline 4 & $\begin{array}{c}122.46 \pm 12.89 \\
(695)\end{array}$ & $\begin{array}{c}126.84 \pm 14.87 \\
i 660)\end{array}$ & $\langle 0.0 \%$ \\
\hline $5+6$ & $\begin{array}{c}12716 \pm 1438 \\
1296)\end{array}$ & $\begin{array}{c}130,62 \pm 13,25 \\
\{279\}\end{array}$ & $<0,00 \mathrm{~s}$ \\
\hline
\end{tabular}

$1=\bar{X} \pm$ D.L. inúmera de casisp pou mituda de próbstos

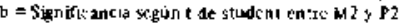

Tabla 4.

Edad promedio (meses) de diferentes etapas de desarrollo mamario en escolares de sexo femenino, por nivel socioeconónico

\begin{tabular}{|c|c|c|c|c|c|c|c|}
\hline $\begin{array}{l}\text { Ftapas dt } \\
\text { Tanner }\end{array}$ & $1+2(A)$ & $3(B)$ & $\begin{array}{c}\text { Nivel Socioceonómico } \\
4 \text { (C) }\end{array}$ & $\begin{array}{l}\text { (Graftar) } \\
3+6(0)\end{array}$ & Total & pb & $\mathbf{p c}$ \\
\hline M2 & $\begin{array}{c}126,78 \pm 14,10^{a} \\
(1 \mathrm{BO} 0)\end{array}$ & $\begin{array}{c}123,73 \pm 17,01 \\
(412)\end{array}$ & $\begin{array}{c}122,46 \pm 17,89 \\
(695)\end{array}$ & $\begin{array}{c}127,16 \pm 14,88 \\
(296)\end{array}$ & $\begin{array}{c}124,53 \pm 17,01 \\
(1573)\end{array}$ & 0,01 & $\begin{array}{l}\text { A/B } 0,05 \\
\text { A/C } 0,001 \\
\text { B/D } 0,01 \\
\text { C/D } 0,001\end{array}$ \\
\hline M3 & $\begin{array}{c}136 \approx 3 \pm 17.32 \\
(137)\end{array}$ & $\begin{array}{c}140,01 \pm 16,33 \\
(281)\end{array}$ & $\begin{array}{c}138,36 \pm 16,52 \\
1498)\end{array}$ & $\begin{array}{c}140,81 \pm 11,83 \\
(214)\end{array}$ & $\begin{array}{c}138,77 \pm 16,13 \\
(1130)\end{array}$ & 0,05 & $\begin{array}{l}\text { A/D } 0,02 \\
C / D 0,05\end{array}$ \\
\hline M4 & $\begin{array}{c}149.62 \pm 17.10 \\
(33)\end{array}$ & $\begin{array}{c}152,6 ? \pm 15,75 \\
(168)\end{array}$ & $\begin{array}{c}152.25 \pm 17,86 \\
(301)\end{array}$ & $\begin{array}{c}154,03 \pm 14,53 \\
(124)\end{array}$ & $\begin{array}{c}152.43 \pm 16,76 \\
(676)\end{array}$ & N.S. & \\
\hline
\end{tabular}

$\mathrm{a}=\overline{\mathrm{X}} \pm$ D.F. (Número de casos) por método de Próbitos

$\mathbf{h}=$ SLgnificancia según prueba $\mathbf{F}$

$c=$ Sipnificancia según $t$ de student

Tablin 5.

Edad promedio (meses) en diferentes etapas de vello púbico en escolares de sexo femenino por nivel sacioeconómico

\begin{tabular}{|c|c|c|c|c|c|c|c|}
\hline $\begin{array}{l}\text { Elapa de } \\
\text { Tanner }\end{array}$ & $1+2(A)$ & $3(B)$ & $\begin{array}{c}\text { Nivel Socioeconómico } \\
4(C)\end{array}$ & $\begin{array}{l}\text { (Graffar) } \\
5+6(D)\end{array}$ & Toul & pb & $\boldsymbol{K}$ \\
\hline P2 & $\begin{array}{c}127,73 \pm 12,52^{a} \\
(178)\end{array}$ & $\begin{array}{c}128,65 \pm 12,50 \\
(387)\end{array}$ & $\begin{array}{c}126,87 \pm 14.87 \\
(660)\end{array}$ & $\begin{array}{c}130,62 \pm 13.25 \\
(279)\end{array}$ & $\begin{array}{c}128,22 \pm 13,69 \\
(1504)\end{array}$ & 0,01 & $\begin{array}{l}\text { A\&D } 0,02 \\
C / D 0,001\end{array}$ \\
\hline P3. & $\begin{array}{c}141,13 \pm 18,20 \\
(118)\end{array}$ & $\begin{array}{c}141,25 \pm 14,27 \\
(273)\end{array}$ & $\begin{array}{c}142,45 \pm 13,91 \\
(441)\end{array}$ & $\begin{array}{c}146,06 \pm 12,92 \\
(176)\end{array}$ & $\begin{array}{c}142,55 \pm 14,43 \\
(1008)\end{array}$ & 0,01 & $\begin{array}{l}\text { AD } 0.02 \\
\text { B/D } 0,001 \\
\text { C/D } 0,005\end{array}$ \\
\hline P4 & $\begin{array}{c}152,52 \pm 18,46 \\
(74)\end{array}$ & $\begin{array}{c}155,96 \pm 14 ; 82 \\
(138)\end{array}$ & $\begin{array}{c}156,37 \pm 15,95 \\
(241)\end{array}$ & $\begin{array}{c}157,90 \pm 14,73 \\
(101)\end{array}$ & $\begin{array}{c}157,33 \pm 17,29 \\
(554)\end{array}$ & 0.05 & $\mathrm{~A} / 00,05$ \\
\hline
\end{tabular}

$a=\overline{\mathrm{X}} \pm$ D.E. (Número de casos) por miétodo de Próbitos

$b=$ Significancia según prueba $F$

c $=$ Signific ancia según $t$ de Studen 1 
El promedio de edad de estas niñas en las diferentes etapas de desarrollo de vello púbico (tabla 5), fue de 128,22 $\pm 13,64$ meses para P2, $142,55 \pm 14,43$ meses para P3 y 157,333 $\pm 17,29$ meses para P4 en el grupo total de escolares. Las escolares de nivel bajo, tienen un promedio de edad significativamente mayor que las de nivel alto para todas las etapas de vello púbico; en tanto que con las escolares de nivel medio bajo, esta diferencia fue significativa sólo en las etapas $\mathrm{P} 2$ y $\mathrm{P} 3$ al compararlas con las de nivel alto.

\section{DISCUSION}

Se analizó el grado de desarrollo puberal alcanzado por 2.307 niñas, representativas de las escolares de la Región Metropolitana de Chile.

Existió un amplio rango de edad entre el inicio y término tanto del desarrollo mamario como del vello púbico. Este hecho que también ha sido descrito en adolescentes europeas y chilenas de clase media, $8,9,21,22$ revela la gran variabilidad que existe con respecto a la edad de presentación y término de la pubertad. Es importante conocer este rango, para hacer un diagnóstico adecuado de pubertad precoz y tardia $y$ evitar estudios costosos en aquellos niños que presentan un comportamiento diferente al promedio de la población. Es probable que estas variaciones puedan deberse a factores de tipo genético y ambiental. Es interesante investigar cual de ellos predomina en los escolares de diferentes NSE. Frish ${ }^{24}$ demostró 2 poblaciones de adolescentes maduradores tardios; en una de ellas la talla final resultó ser significativamente más baja $y$ en esa población los parámetros antropométricos sugirieron la presencia de factores ambientales, en tanto que en los que terminaron con talla normal se sugirió factores de tipo constitucional.

La iniciación más temprana del desartollo mamario que el púbico en una proporción impor. tante de estas niñas y en la población total, al analizar la edad promedio de inicio de ambos eventos por método de próbitos, ocurrió sólo a expensas de las escolares de los NSE medio y bajo, pues en las de NSE alto ambos eventos se produjeron casi simultáneamente. $\mathrm{Si}$ consideramos el desarrollo mamario, las escolares de los NSE medios inician antes la pubertad que las de los NSE alto y bajo. Esta menor edad promedio de botón mamario en las escolares de njvel medio podria estar traduciendo factores genéticos que no se hubiesen manifestado en las de nivel alto, tal vez por mayor impacto de factores de tipo nutricional. Valenzuela y $\operatorname{cols}^{22}$ han sugerido factores raciales en el adelanto de la pubertad detectado en escolares chilenos de clase media y dimorfismo sexual con respecto al crecimiento durante la pubertad, al compararlos con los escolares europeos. Con respecto al desarrollo del vello púbico también se encontraton diferencias significativas por NSE: las escolares de nivel bajo tienen mayor edad al inicio y término del desarrollo del vello púbico, alcanzando diferencias significativas con las de nivel alto en todas las etapas. Esto podria traducir factores ambientales ya que las escolares de NSE bajo tienen menores pesos, tallas y áreas grasa y magra to que sugiere deficit nutricionales, al menos relativos, que pudiesen asociarse a retraso en la aparición de los eventos puberales.

Podemos decir que las escolares de sexo femenino de la Región Metropolitana, tienen un rango de variación de casi 7 años en la aparición de los signos de desarrollo puberal tanto en el inicio del desarrollo mamario como del vello púbico, siendo más temprana la aparición del botón mamario en las escolares de NSE medio y más tardía la del vello púbico en las escolares de NSE bajo, desfasándose así ambos eventos en las adolescentes de clase media y baja, hecho que no se observó en los niveles altos, lo que tal vez podría traducir factores genéticos.

\section{RESUMEN}

Se estudiaron algunas caracteristicas de la pubertad en un corte transversal de 2.328 escolares de sexo femenino, representativas de la Región Metropolitana y de los diferentes niveles socioeconómicos. Se detectó un rango de variación de 96 a 183 meses de edad en la iniciación del desarrollo mamarjo y de 100 a 183 en el vello púbico. Se encontró una diferencia significativa entre la iniciación del desarrollo mamario y del vello púbico, sólo en las escolares de niveles socioeconómicos medios $y$ bajos, ocurriendo más temprano el desarrollo mamario (123,7 y 122,4 vs 128,6 y 126,8 meses respectivamente). Se sugiere que es posible que factores ambientales $y$ genéticos estén involucrados en estos hallazgos. 


\section{REFERENCIAS}

1. Shender I.R., Nissbaum M.. Kaplan E.: Dclayed puberty and short stature in adolescents. Pediats Ann 1978: 7: 605-613.

2. Smith D.W.: Approach to the diagnosis, classilication and nature of growth deficiency disarders. In growth and its disorders Vol VX in the series "Major problems in tinjical pediatrics" Ed. Smith D.W., Philadelphia, W.B. Saunders Co. 1977; 62-69.

3. Beas $F$., Contreras L., Macciont A., Lacassie 1. Monkeberg F., Muzzo S.: Diagnóstico del retardo del crecimicnto en el niño. Rev Chil Pediatr 1968: 39: 649-680.

4. Beck W. Wuttke W.: Diurnal variations of plasma luteinizing homone, follicle stimulation hormone, and prolacin in boys and girls trom birth to puberty. J Clin Endocinol Metab 1980; 50: $635-639$

5. Kulin H.E., Grumbach M.M., Kaplan S.L.: Changing sensitivity of the pubertal gonadal hypothalamic fecdback mechanism in man. Science 1969: 166: 1012-1013.

6. Duchorme J.R., Collu R.: Pubertal development: normal. precocius and delayed. Clin Fndocrinol Metab 1982;11: 57-83.

7. Sizonenko P.C.: Endocrinology in prtadolescents and adolescents. Am J Dis Child 1978; 132: 704-712.

8. Marshall W.A., Tanner J.M. Variations in pattem of puberal changes in girls. Arcli Dis Clijld 1969: 44 $291-303$

9. Belmaker E.: Sexual maturation of Jerusalem schoolgirls and its association with socio-economic factors and ethric group. Am Human Biol 1982; 9: $321-328$.

10. Frish R.E.: Body fat, puberty and fertility. Biol Rew 1984;59: 161-188.

11. Frish R.E.: Critical weight at menarchs, initiation of the adolescent growth spurt, and control of puberty. In "Control of the onset of puberty" Grumbash, Grene and Mayer. Wiley. New York pp. $1974 ; 403-423$.

12. Kennedy G.C. Mitra J.: Body weight and tood intake as initiating factors for puberty in the rat. J
Physiol $1963 ; 166: 408-418$.

13. Frish $R$, Revelte $R$.: Variation in body weight and the age of the adolescent growth spurt among Latin American and Asian Population in relation to calorie supplics. Human Biol 1965: 41: 185-212.

14. Beaumont P.J., George C.W. Pimstone, B.L., Winik A. I. : Body weight and pituitary response to hy pothalamic reicasing hormones in patjents with anorexid nervosa. J Clin Endocrinol Metab 1976; 43: 487-496

15. Bogar R.M. Katz J., Finketstein J.W., Xaren S., Weiner $H$. Weltzmon: Inmaturity of the 24 hour luteinifing homone secretory pattern. New knkland I of Med 1974;291:864-865.

16. Lopez C.M., Tovar E.G., Farid C.N. Landaeta J.M., Méndez C.H.: Estudios comparados de la estatura $y$ edad de la menarquia según estrato socjocconómico en Venezuela. Arch Latinoam Nutr 1981; 311: $740-757$.

17. Lee M.M.C., Chong K.S.F., Chan M.M.C.: Sexual maturation of chinese gir ls in Hong Kong. Pediatrics 1963:32: 389-396.

18. Patri A.M., Valenzuela Y.C., Morales C.L., Saavedra Z.J. Figueroa O.L.: lidad de la Menarquía y lactores determinantes. Cuad Med Soc 1980: 12-20.

19. Zasharias L., Wurtman R.J., Schatzaff M.: Sexual maturation in contemporary American girls. Am J Obstet Gynecol 1970; 108: 833-846

20. Alvarez $M$. Wurgaft $F$.. Salazar M.: Mediciones del nivel socioeconomico bajo urbano en familias con lactantes desnutridos. Arch Latinoam Nutr 1982. 32: $650-662$

21. Avéndaño B.A., Patri M.A., Valenzuela Y.C.: Antropometr ía de Fiscolares Fiscales del Area Metropolitana Norte de Stgo. Ill Parte. Maduración sexual. Cuad Med Soc 1975: 16: 17-24.

22. Valenzuela Y.C., Avendaño A.R.: Antropometría y maduración de escolates de un Area de Stgo. de Chile. Bol Of Sanit Panam 1979; 87 (2): 113-131.

23. Rona $R$.: Secular trend of pubertal development in Chile. I of Human End 1975;4: 251-257.

24. Frisch R.E., Rewelle R.: Height weight of girls and hays at the time of peak velocity of growth in height and weight. Longitudinal data. Hum Biol $1969 ; 41: 536.599$. 